\title{
LONG LOOP AND SPINAL REFLEXES IN MAN DURING PREPARATION FOR INTENDED DIRECTIONAL HAND MOVEMENTS
}

\author{
MICHEL BONNET ${ }^{1}$ AND JEAN REQUIN \\ Department of Experimental Psychobiology, Institute of Neurophysiology and Psychophysiology, National Center for \\ Scientific Research, Marseilles, France
}

Received February 6, 1981; Revised August 3, 1981; Accepted September 9, 1981

\begin{abstract}
Advance information about the direction of a movement to be performed in a reaction time (RT) paradigm is known (1) to improve performance, (2) not to result in any related change in monosynaptic spinal reflexes triggered at the time of the response signal, and (3) to trigger related changes in the activity of a number of neurons in the motor cortex.

The aim of this experiment was to compare the evolution of the early (M1) and late (M2) responses to a muscular overload during the foreperiod of an RT task, according to advance information about movement direction.

Four subjects were trained to perform wrist extension and flexion movements in order to point to a visual target on either the right or left of a display panel. One second before the movement signal, a warning signal indicated to the subject at which target he was to point. A hand position perturbation causing a stretch of the wrist flexors was triggered at various unpredictable times during this 1-sec foreperiod.

The analysis of the surface electromyogram recorded upon wrist flexors showed that the evolution of M1 and M2 response amplitudes differed during the foreperiod. M1 increased up to 600 msec after the warning and then decreased until the response signal, while M2 continued to increase during the whole foreperiod. There was no change, however, in this differential evolution in relation to advance information about movement direction.

The results were interpreted as the combination of two preparatory processes: first, a general activation, expressed by M2 increase, which can be viewed as a pre-setting of motor structures for a rapid execution of the motor program, and second, an inhibition of spindle afferent action upon spinal motor structures, expressed by the decrease of M1 response before movement execution, which can be viewed as a mechanism for protecting the motoneurons about to be activated from peripheral control pathways. Lastly, no specific pre-setting of the motor structures involved in M1 and M2 electromyographic responses according to the movement direction was found.
\end{abstract}

An examination of spinal excitability changes in human subjects by soliciting the monosynaptic reflex either by electrical stimulation of the nerve or by tendinous tap has shown that, during the foreperiod of reaction time (RT) tasks, there is a selective modification in the excitability of the motoneurons controlling the muscle involved in the motor response (Requin, 1969; Gerilovski and Tsekov, 1971; Requin and Paillard, 1971). However, this pre-setting was expressed in a relative depression of reflex pathway reactivity, the depth of which showed no change whether the muscle was acting as agonist or

\footnotetext{
${ }^{1}$ To whom correspondence should be addressed at Department of Experimental Psychobiology, Institute of Neurophysiology and Psychophysiology 3-B.P. 71, National Center for Scientific Research, 13277 Marseille, Cedex 9, France.
}

antagonist nor when the force required to perform the intended movement was varied (Gerilovski and Tsekov, 1972; Requin et al., 1977). A possible explanation for this set of data was that the efficiency of the reflexogenic stimulation was decreased during motor preparation by an inhibitory phenomenon spreading to all of the spinal structures involved in motor activity, the functional meaning of which might be related to the need existing at that moment to protect motoneurons against disturbing inputs (Requin, 1980; Requin and Paillard, 1971; Requin et al., 1977).

The same lack of specificity in changes of monosynaptic reflex amplitude during preparation for antagonistic movements was observed when a muscular overload was used to trigger a stretch reflex in arm muscles (Hammond, 1960; Crago et al., 1976; Evarts and Granit, 1976). 
As far as we know, only two unrepeated observations, showing a relative enhancement of monosynaptic stretch reflex when the muscle had to be activated to perform the movement, disagree with this set of negative data; the first was by Evarts and Tanji (1974) on one monkey and the other was by Hagbarth (1967) in an experiment where sequential effects were an alternative explanation for the results observed, as underlined by Evarts and Granit (1976). Thus, the hypothesis that there is a presetting of spinal motor structures during preparation for action, resulting in monosynaptic reflex changes closely related to movement parameters or at least strictly focused on the muscle to be activated, has yet to be confirmed.

On the other hand, the activity of the pyramidal tract neurons of the motor cortex in monkeys was found to be modified according to the instructions as to movement direction provided by a warning signal, sometimes as early as $200 \mathrm{msec}$ after this signal (Tanji and Evarts, 1976). Although these changes in cortical activity were not accompanied by any change in the tonic electromyographic (EMG) activity of the muscle involved in the motor task, they strongly suggested that movement direction can be specified during preparatory processes preceding motor execution. This hypothesis prompted us to try to detect this specification of movement direction at the cortical level during the preparatory period of an RT paradigm in human subjects.

Since it is known that successive EMG responses (M1, M2, and M3) can be triggered either by a muscular overload (Lee and Tatton, 1975) or by an electrical stimulation of spindle afferents (Milner-Brown et al., 1975), cortical motor structures must be considered to be accessible for an experimental study in man. However, although a number of studies exist in this field (for review, see Desmedt, 1978), the "transcortical" feature of pathways underlying these late (M2 and M3) responses is still questionable. For instance, Ghez and Shinoda (1978) have shown in the spinal cat that these responses can involve the spinal pathway only. This result was claimed again by Tracey et al. (1980) in the spinal monkey without any clear experimental evidence, whereas North and Tatton (1980) have emphasized the differences observed between species: "In the cat, motor cortical neuron (MCN) activity does not contribute to the generation of the 2nd EMG response peak, in support of the transection studies and in contrast to MCN activity related to the M2 response for monkey distal upper limb musculature." Lastly, from their recent studies on man, Hagbarth et al. (1981) have claimed that the segmented EMG responses are almost solely due to segmented afferent burst rather than multiple central reflex pathways.

This "spinal" interpretation is not supported, however, by studies showing that the strong depressive effect of muscular vibrations acts selectively on the M1 response but not upon the later responses (Hendrie and Lee, 1978), contrary to what Hagbarth's view would suppose. Along the same lines, Milner-Brown et al. (1975), by using a brief transcutaneous electrical stimulation of the motor nerve, which results in one Ia burst only, observed the segmented EMG responses and pointed out that these were time-locked to the surface potentials recorded over the cervical spinal cord and the hand area of the motor and somatosensory cortex.

Finally, clear evidence for a cortical involvement in the M2 response triggered by a spindle activation was provided by Chauvel et al. (1978) and Tatton et al. (1975) in the monkey and by Marsden et al. (1978) in man. As matters stand at present, there is thus some agreement with the view of Evarts and Vaughn (1978) interpreting the successive EMG responses as being first, the monosynaptic stretch reflex response (M1); second, the linked involvement of spinal and supraspinal reflex arcs (M2); and third, the first stage of the intended response (M3) which was not seen to depend on stimulation parameters.

However, in the study of preparation for movement, the paradigm classically used to point out pre-setting processes seemed unsuitable because hand or arm perturbation causing stretching of the muscle was used at the same time as a test of the effect on nervous structures of instructions given to the subject and as an imperative signal to perform this instructed response. The crucial point here is that the meaning of changes in response amplitude observed under these conditions is not clear because it is difficult to decide whether they express fluctuations in the functional state of structures still "waiting for" the imperative signal or whether they already underlie the first stages of the responding process itself.

In the present experiment, we studied in man EMG responses to overload in a simple RT task with a foreperiod of fixed duration and a warning signal providing advance information about movement direction. Experimental conditions differed from those used by Evarts and Tanji (1974) on the monkey in two ways. First, the signal triggering the intended response was different from the stimulus triggering the EMG responses. Secondly and consequently, M1 and M2 responses were studied not only at the time of the response signal but also during the whole foreperiod between the warning signal and the response signal.

\section{Materials and Methods}

Apparatus. The subject sat in front of a table to which his right forearm was attached in a restraining device. The palm and fingers of his open hand were pressed against a vertical plate which can rotate in the horizontal plane around a vertical axis identical with the wrist axis. On the distal part of the plate was fixed a spring, the action of which pulled the plate to the right, thus involving a passive extension of the hand. Therefore, to keep his hand steady, the subject had to activate the wrist flexors constantly in order to counterbalance the spring force (1.8 Newton $\times$ meter). One meter in front of the subject, three light-emitting diodes were arranged horizontally on a vertical display panel-in the center, on the left, and on the right, respectively $-5 \mathrm{~cm}$ apart. They served as visual signals and as targets for pointing movements: a rod extending the plate moved by the subject's hand made the pointing task easy, as the rod end simply had to be brought in line with the target. Pointing from the central target to the left one involved a $5^{\circ}$ angular displacement of the hand produced by a synergistic activation of the wrist flexor muscles. Pointing to the right 
target involved a hand displacement of the same extent in the other direction produced by relaxing the flexor muscles and by activating the antagonistic wrist extensors. In order to measure reaction time (RT) for these pointing movements, two photo cells, placed $1 \mathrm{~cm}$ to the left and right of the central target, were darkened when a displacement of the rod occurred as the subject was moving it from the central target to a lateral one. On the left side of the hand plate, an electromagnetic hammer was installed in such a way that when the subject was pointing to the central target, the hammer could be activated to tap the distal part of the plate, causing a perturbation of the hand position toward the right and thus a sudden stretch of the wrist flexor muscles.

Procedure. Successive events of a trial, controlled by a PDP 12 computer, were as follows (cf., Fig. 1). First, the illumination of the central target, as a pre-positioning signal, required the subject to point centrally and to maintain this position steadily although the stretched spring was pulling his hand to the right. After $2 \mathrm{sec}$, the central target was turned off and one of the two lateral targets was illuminated for $50 \mathrm{msec}$, instructing the subject in advance about the direction of the pointing movement that he would have to perform. This warning signal started a preparatory period of $1 \mathrm{sec}$, during which an activation of the hammer perturbing the hand position occurred after an unpredictable delay. The preparatory period was ended by again illuminating the central target for $50 \mathrm{msec}$, as a response signal, triggering the previously instructed pointing movement to the corresponding lateral target. After $10 \mathrm{sec}$, a new trial started.

Experimental design. During a series of trials, the warning signal was distributed randomly between the two lateral targets with a 0.50 probability for each of them. The probability for the response signal to occur, by illuminating the central target, was 0.88 . The remaining trials, where the response signal did not occur, were included randomly within a series, as catch trials, in order to prevent anticipatory responses, which could appear in such a simple RT task with a preparatory period of fixed duration. The position of the mechanical impulse disturbing the hand position was varied randomly from the warning signal to the response signal by steps of 200 msec. In a series of 56 trials, each step was tested eight times, except at the time of the warning signal when hand perturbation was triggered in 16 trials, in order to provide a suitable reference level for assessing changes in reflex responses during the preparatory period. Each subject performed the task during four successive daily sessions, with each session consisting of four identical series of trials. These four experimental sessions were preceded by a training session.

Subjects, instructions, and training. Four righthanded subjects participated in this experiment. After a brief explanation about the apparatus and general procedure, they were instructed to point as precisely as possible to the central target when it was illuminated as a pre-positioning signal, to maintain this position when one of the lateral targets lighted up as a warning signal, and to point as fast and as accurately as possible to this target when the central target lighted up again as a response signal. The subject also was informed that hand position would be briefly disturbed during the task, but no information was given about the precise meaning of this event which he was to ignore as far as possible.

During the training session, subjects were instructed (a) to point to the central target as accurately as possible in order to keep steady the hand under which the me-

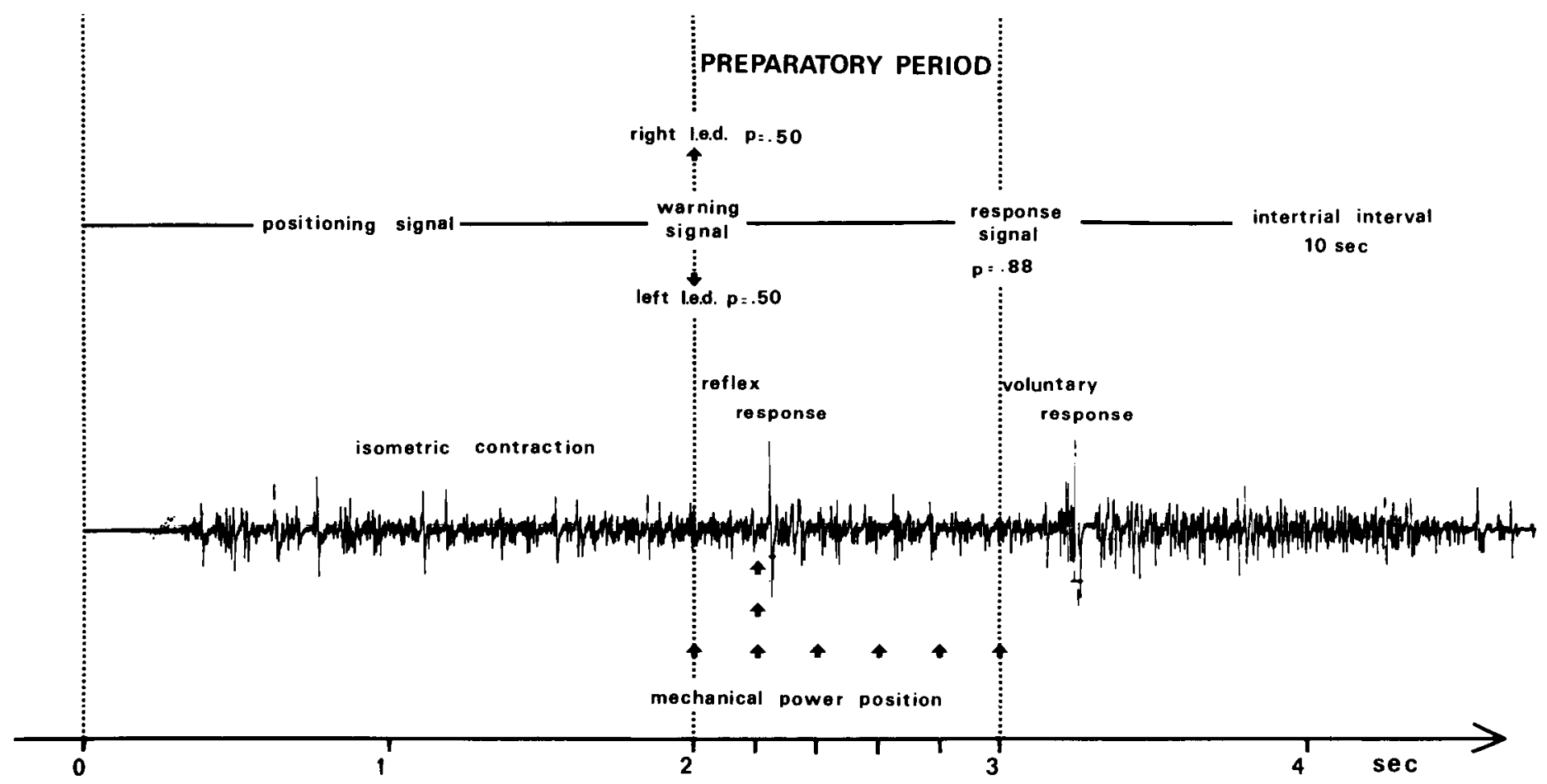

Figure 1. The timing of one trial. l.e.d., light-emitting diode. 
chanical stimulation was applied (a maximum allowance of $0.5^{\circ}$ in the rotation angle, i.e., $5 \mathrm{~mm}$ on the display panel, was adopted as a training criterion) and (b) to compensate for the enforced hand displacement as fast as possible in order to point again to the central target when the response signal occurred. After training, this return of the hand became an automatic reaction and was performed as quickly as $300 \mathrm{msec}$ without any overshoot.

Recordings. Two usual surface Ag-AgCl electrodes, 8 $\mathrm{mm}$ in diameter, were fixed on the inside of the right forearm upon the flexor carpi radialis, $2 \mathrm{~cm}$ apart, $5 \mathrm{~cm}$ from the elbow joint. Electromyographic activity was amplified and then transmitted to a special digital voltmeter in order to measure the amplitude of M1 and M2 responses triggered by hand perturbation. At each session, for the first series of trials, which was a training series, the data provided were not included in the final computation of results but were used to check the mean latency of both M1 and M2 responses for each subject in order to adjust two electronic windows, $20 \mathrm{msec}$ in width, during which the voltmeter measured the peak-to-peak amplitude of both responses. In most cases, these windows were adjusted from 20 to $40 \mathrm{msec}$ for $\mathrm{Ml}$ and from 45 to $65 \mathrm{msec}$ for M2.

In a pilot experiment, a comparison was made between the data obtained with this peak-to-peak method, the inaccuracy of which can result from the possibly polyphasic shape of M2, and the data obtained from the EMG integration during the period defined by the electronic window, the inaccuracy of which can result from the slight M2 latency changes and/or the integration of basic EMG activity. No significant difference was found. Moreover, the fact that, under our experimental condition, M1 and M2 responses were well synchronized, as can be seen in Figure 2, made it easy to apply the peakto-peak measurement procedure classically used in the study of monosynaptic reflex responses.
Results

\section{Response triggered by hand perturbation}

Figure 2 shows three samples of EMG activity following hand perturbation (left) and three samples of EMG activity averaged over 56 trials (right). Three successive synchronous EMG activities can be observed, which were identified as M1, M2, and M3 responses, according to the classification proposed by Tatton et al. (1975).

$M 1$ response. The first, $\mathrm{M} 1$ response, which appeared after a 25-msec fixed latency, was biphasic in shape and looked very similar to the classical monosynaptic stretch reflex triggered by a tendinous tap.

$M 2$ response. The second, $\mathrm{M} 2$ response, with a latency of about $55 \mathrm{msec}$, was not as steady in latency and shape as M1. It should be noted that, during the training session, we did not observe any of the latency change described by Burke et al. (1980) triggered by whether the subject was informed about overload occurrence.

M3 response. The third, M3 response appeared as an often polyphasic EMG activity between 80 and $125 \mathrm{msec}$ after hand perturbation. These responses, which could result from the activation of long transcerebellar and/or transcortical loops, were not observed in all of the subjects (as already underlined by Milner-Brown et al., 1975) and, consequently, were not taken into account.

On the other hand, no systematic changes in basic EMG activity were observed during the preparatory period. This is not surprising since, under our experimental conditions, any change in the wrist flexor muscle activation would have resulted in a change in the hand position that the subject was especially trained to hold steady.

Time course of $M 1$ and $M 2$ response amplitude during preparatory period

Before averaging the data over series, sessions, and subjects, the amplitude of responses observed during the foreperiod for each series of trials was changed to $Z$
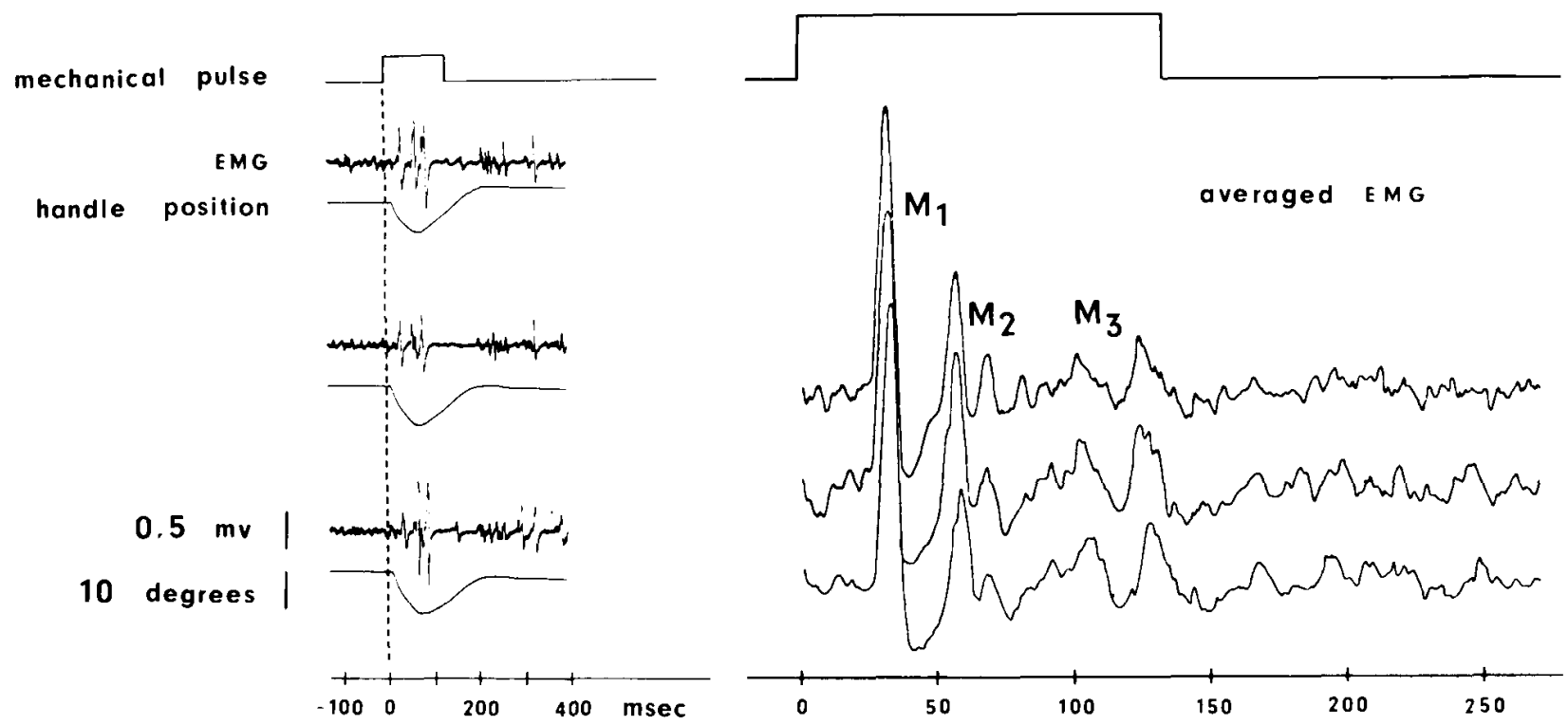

Figure 2. Samples of EMG responses triggered by a rapid stretch of the wrist flexors. Left, Samples of EMG responses and handle position changes recorded on the same subject during three trials in the training session. Right, Averaged recording (another subject) of the negative phase of EMG activity during three successive experimental series of 56 trials. 


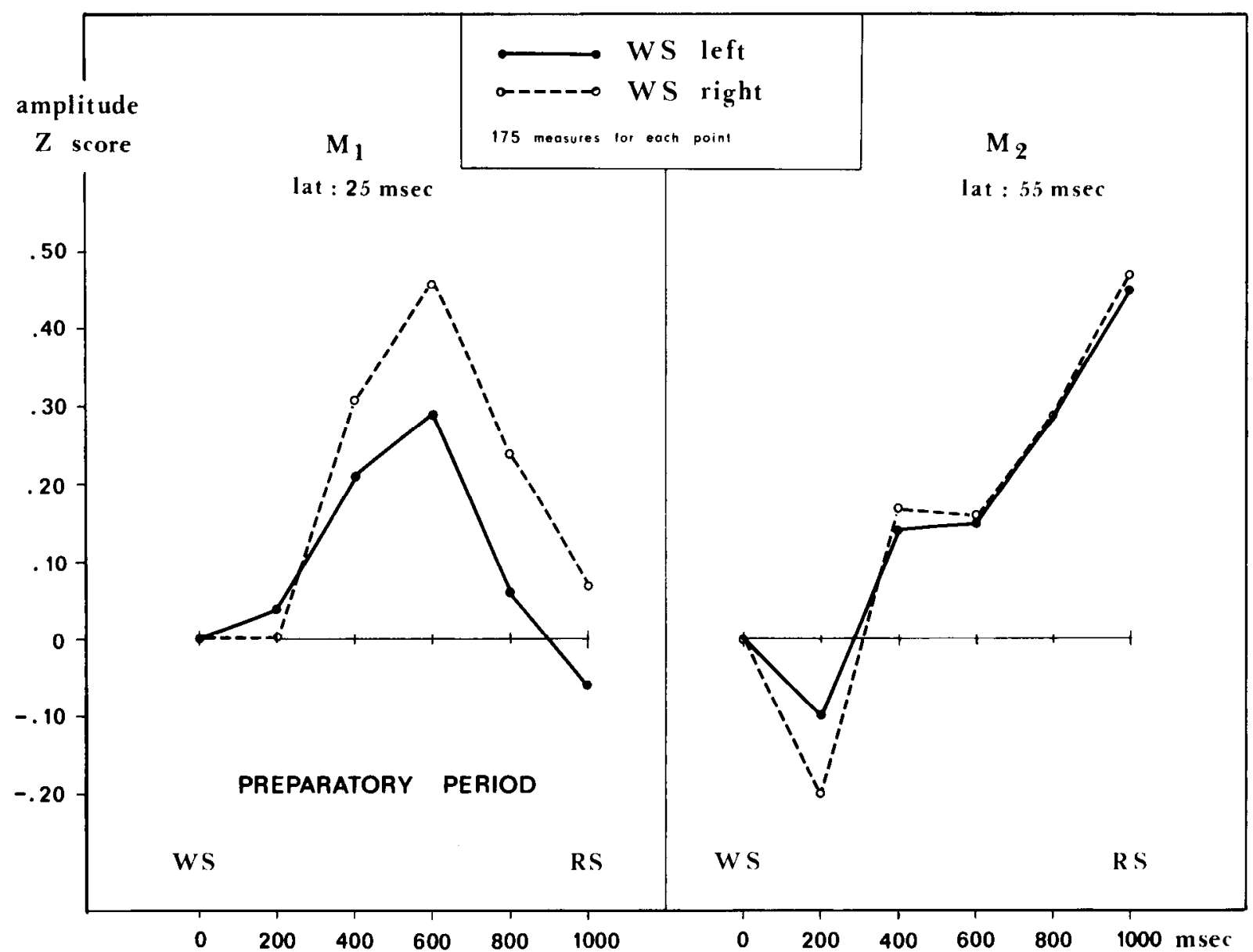

Figure 3. Time course of M1 and M2 response amplitude expressed by $Z$ scores (see explanations in the text) from the warning signal ( WS ) to the response signal $(R S)$. Data are separated according to prior instructions given to the subject by the WS about hand movement direction, either to the left by activating wrist flexor muscles or to the right by relaxing these muscles. The data are averaged for four subjects during four sessions. lat, latency.

scores, the ratios of the standard deviation of the distribution of response amplitudes observed when the hand perturbation was triggered at the time of the warning signal, thus considered as a reference amplitude level.

Figure 3 shows the mean amplitude of M1 and M2 responses according to the time when the hand perturbation occurred during the foreperiod. Data are separated according to the information given to the subject by the warning signal about the direction, right or left, of his pointing movement.

The analysis of variance presented in Table I confirms that both M1 and M2 response amplitudes differed significantly among subjects $(F=6.01 ; d f=3 ; p<0.01)$ and showed significant changes during the foreperiod $(F=$ $9.30 ; d f=4 ; p<0.01)$. The time course of these changes was clearly different for $\mathrm{M} 1$ and $\mathrm{M} 2$ responses $(F=8.70$; $d f=4 ; p<0.01$ ): M1 amplitude increased (to $14 \%$ of its control value on average) until $600 \mathrm{msec}$ after the warning signal and then decreased until the end of the foreperiod, reaching, at the time of the response signal, a level similar to that observed at the time of the warning signal; M2 amplitude, after a brief and slight decrease 200 msec following the warning signal, increased continuously during the foreperiod, peaking at the time of the response signal. This maximum increase was $25 \%$ of the control level on average.
'TABLE' I

Analysis of variance of $M 1$ and $M 2$ reflex amplitude during the preparatory period preceding the execution of directional movements

\begin{tabular}{lcccc}
\hline \multicolumn{1}{c}{ Source of Variation } & $\begin{array}{c}\text { Degrees of } \\
\text { Freedom }\end{array}$ & $F$ Ratio & $\begin{array}{c}\text { Level of Sig- } \\
\text { nificance }\end{array}$ \\
\hline $\begin{array}{l}\text { Subjects }(n=4) \\
\text { Type of response } \\
\quad \text { M1 or M2) }\end{array}$ & $S$ & 3 & 6.01 & $p<0.01$ \\
$\begin{array}{l}\text { Advance information } \\
\quad \text { (right or left) }\end{array}$ & $I$ & 1 & 2.76 & NS \\
$\begin{array}{l}\text { Mechanical stimulation delay } \\
\quad(200,400,600,800, \text { or 1000 }\end{array}$ & $D$ & 4 & 9.30 & $p<0.01$ \\
$\quad$ msec) & & & & \\
& & & & \\
Interactions & & & & \\
$\quad R \times I$ & & 4 & 3.27 & $\mathrm{NS}$ \\
$R \times D$ & & 4 & 0.70 & $p<0.01$ \\
$I \times D$ & & 4 & 0.15 & $\mathrm{NS}$ \\
$R \times I \times D$ & & & & $\mathrm{NS}$ \\
\hline
\end{tabular}

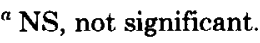

\section{Effect on response amplitude of advance information} about movement direction

The mean amplitude of both responses during the foreperiod did not depend upon the information given to the subject by the warning signal $(F=2.76 ; d f=1$; not 
significant), and there was no significant difference between the effects of instructions on M1 and M2 ( $F=$ 3.27 ; $d f=1$; not significant), although there was a tendency for M1 amplitude to be lower when the left warning signal instructed the subject to activate his forearm flexor muscles. This lack of a differential warning signal effect was observed during the whole foreperiod $(F=0.55 ; d f$ $=4$; not significant) and was similar for both responses ( $F=0.15 ; d f=4$; not significant).

\section{Discussion}

First of all, the different time course of M1 and M2 responses during the foreperiod must be stressed. The evolution of M1 amplitude can be considered to be very similar to what we previously observed on monosynaptic reflexes triggered in the soleus muscle: after an initial increase, peaking later in the arm $(600 \mathrm{msec})$ than in the leg (about $300 \mathrm{msec}$ ), there was a progressive decrease in response amplitude until the response signal. This second decreasing phase did not appear in the M2 response, the amplitude of which increased continuously during the foreperiod. These results suggest that two different central processes affect motor structures when preparing to control a movement: ( $a$ ) an activation process, expressed by $\mathrm{M} 2$ amplitude evolution and by the first phase of $\mathrm{M} 1$ amplitude increase, showing a progressive enhancement of the excitability of motor structures, or at least of motoneurons, which fits well with the classical hypothesis of a pre-setting process facilitating motor response performance by reducing the time necessary for cortical structures to activate motoneurons; $(b)$ an inhibitory process, expressed by the decrease of M1 amplitude in the second part of the foreperiod, which could be caused, as we have already suggested (Requin and Paillard, 1971; Requin et al., 1977), by a presynaptic mechanism acting on primary muscular afferents, since the depression of the monosynaptic pathway reactivity shown by M1 appears simultaneously with an increase in the reactivity of a more complex pathway, also ending in motoneurons, however, as shown by M2. In regard to the activation process, since Burke et al. (1980) did not observe any change in spindle activity during preparation for movement, it is likely that this facilitation did not result from an increase in $\gamma$ motoneuron activity.

The second important finding is that advance information about movement direction (i.e., about the alternative for the muscle to either increase or decrease its prior activation) did not result in any change in the time course of either M1 or M2 response amplitude. Concerning $\mathrm{M} 1$, this result confirms what we have always observed previously with monosynaptic reflexes triggered in the soleus muscle under similar task conditions (Requin et al., 1977). The inhibitory phenomenon depressing the monosynaptic reflex pathway just before movement is not limited to motoneurons about to be activated but applies more widely to spinal structures, at least to those involved in moving the same joint, even at the whole segmental level. In the same way, the lack of change in M2 response according to advance information provided about movement direction thus could strengthen the conclusion that the pre-setting of spinal motor structures does not focus on the motoneuron pool directly responsible for movement performance. By overspreading a number of nervous structures, at least at the spinal level, this reactivity enhancement seems more likely to reflect some unspecific activation phenomenon. One can suggest, for instance, that nervous structures highly sensitive to general arousal which underlie motor preparation are included in the nervous pathways through which this late $\mathrm{M} 2$ response is expressed.

However, before concluding, the lack of any differential effect of instructions on M2 amplitude must be discussed in relation to the data collected on the monkey by Tanji and Evarts (1976), who failed to show any effect on tonic EMG activity of the differential discharge frequency changes observed in a number of pyramidal tract neurons connected to the motoneurons which control the muscles involved in the task. In the framework of a "transcortical" interpretation of $\mathrm{M} 2$, these changes in motor cortex activity lead one to expect M2, which was not recorded by Tanji and Evarts, to be sensitive to prior instructions. However, before claiming that our results do not support this expectation, some differences between the experimental conditions set up in the two studies must be underlined. In Tanji and Evarts's experiment, speed requirements were given priority in movement execution, since the central pointing area was as wide as $10^{\circ}$ and the correct "push" and "pull" responses were defined only by a lever displacement of more than $15^{\circ}$ beyond this central area but without any pointing at a target of limited width. On the contrary, accuracy requirements were made crucial in our experimental conditions, since not only the central target width was reduced to $0.5^{\circ}$ but this accuracy constraint also was applied to lateral pointing movements. Thus, one can suggest that the former conditions were especially likely to involve differential pre-setting according to prior instructions, since the response movements were characterized mainly by either an agonistic or an antagonistic muscular activity only, whereas, under the latter conditions, both response movements possibly involve some co-activation, and thus some co-pre-setting, in the precise control exerted on movement extent.

On the other hand, in our experiment, this lack of differential effect of prior instructions on M2 also could be related to the fact that hand position was always disturbed in the same direction: if one supposes that the subject was expecting not to perform the instructed movement but to resist overload, it would be possible that he was always preparing to activate his wrist flexors. This objection should probably be rejected, however, first, because the mean RT observed for flexion (273 $\mathrm{msec})$ and for extension $(270 \mathrm{msec})$ clearly indicates that the subjects were well prepared for performing both response movements as quickly as possible and, second, because it has been shown previously by Thomas et al. (1977) that M2 response amplitude was not changed when the direction of hand perturbation was made unpredictable.

In conclusion, this discussion stresses the role possibly played by biomechanical requirements of the motor task as well as cognitive aspects of the experimental conditions under which this task is performed. Both will have to be checked carefully in further studies of the cortical and subcortical mechanisms underlying preparatory processes for motor activity. 


\section{References}

Burke, D., B. McKean, M. F. Skuse, and R. A. Westerman (1980) Anticipation and fusimotor activity in preparation for a voluntary contraction. J. Physiol. (Lond.) 306: 337-348.

Chauvel, P., J. Lauvel, and M. Lamarche (1978) Transcortical reflexes and focal motor epilepsy. Electroencephalogr. Clin. Neurophysiol. 45: 309-318.

Crago, P. E., J. C. Houk, and A. Hasan (1976) Regulatory actions of human stretch reflex. J. Neurophysiol. 39: 925-936.

Desmedt, J. E., ed. (1978) Cerebral Motor Control in Man: Long Loop Mechanisms, Karger, Basel.

Evarts, E. V., and R. Granit (1976) Relations of reflexes and intended movements. Prog. Brain Res. 44: 1-14.

Evarts, E. V., and J. Tanji (1974) Gating of motor cortex reflexes by prior instruction. Brain Res. 71: 479-494.

Evarts, E. V., and W. J. Vaughn (1978) Intended arm movements in response to externally produced arm displacements in man. In Cerebral Motor Control in Man: Long Loop Mechanisms, J. E. Desmedt, ed., pp. 178-192, Karger, Basel. Gerilovski, L., and T. Tsekov (1971) Amplitude changes of Hreflex during a fixed waiting period as a function of absolute probability of the response signal. In Visual Information Processing and Control of Motor Activity, pp. 397-401, Bulgarian Academy of Science, Sofia, Bulgaria.

Gerilovski, L., and T. Tsekov (1972) Amplitude changes of the $\mathrm{H}$-reflex during a fixed waiting period when the tested muscle is agonist, antagonist or does not take part in movements. Agressologie 13: 31-35.

Ghez, C., and Y. Shinoda (1978) Spinal mechanisms of the functional stretch reflex. Exp. Brain Res. 32: 55-68.

Hagbarth, K. E. (1967) EMG studies of stretch reflexes in man. In Recent Advances in Clinical Neurophysiology, L. Widen, ed., pp. 74-79, Elsevier, Amsterdam.

Hagbarth, K. E., J. V. Häggland, E. Y. Wallin, and R. R. Young (1981) Grouped spindle and EMG responses to abrupt wrist extension movements in man. J. Physiol. (Lond.) 312: 81-96.

Hammond, P. H. (1960) An experimental study of servo action in human muscular control. Proceeding of the 3rd International Congress on Medical Electronics, London, pp. 190199.

Hendrie, A., and R. G. Lee (1978) Selective effects of vibration on human spinal and long-loop reflexes. Brain Res. 157: 369375.

Lee, R. G., and W. G. Tatton (1975) Motor responses to sudden limb displacements in primates with specific CNS lesions and in human patients with motor system disorders. Can. J.
Neurol. Sci. 2: 285-293.

Marsden, C. D., P. A. Merton, H. B. Morton, and J. Adam (1978) The effect of lesions of the central nervous system on long-latency stretch reflexes in the human thumb. In Cerebral Motor Control in Man: Long Loop Mechanisms, J. E. Desmedt, ed., pp. 334-341, Karger, Basel.

Milner-Brown, H. S., R. B. Stein, and R. G. Lee (1975) Synchronization of human motor units: Possible roles of exercise and supraspinal reflexes. Electroencephalogr. Clin. Neurophysiol. 38: 245-254.

North, A. G. E., and W. G. Tatton (1980) Temporal relations between motor cortical and electromyographic responses to imposed forelimb displacements in the cat. Soc. Neurosci. Abstr. 6: 392.

Requin, J. (1969) Some data on neurophysiological processes involved in the preparatory motor activity to reaction time performance. In Attention and Performance II, W. G. Koster, ed., pp. 358-367, North-Holland Publishing Co., Amsterdam.

Requin, J. (1980) Toward a psychobiology of preparation for action. In Tutorials in Motor Behavior, G. E. Stelmach and J. Requin, eds., pp. 373-398, North-Holland Publishing Co. Amsterdam.

Requin, J., and J. Paillard (1971) Depression of spinal monosynaptic reflexes as a specific aspect of preparatory motor set in visual reaction time. In Visual Information Processing and Control of Motor Activity, pp. 391-396, Bulgarian Academy of Science, Sofia, Bulgaria.

Requin, J., M. Bonnet, and A. Semjen (1977) Is there a specificity in the supraspinal control of motor structures during preparation? In Attention and Performance IV, S. Dornic, ed., pp. 139-174, Lawrence Erlbaum Associates, Hillsdale, NJ.

Tanji, J., and E. V. Evarts (1976) Anticipatory activity of motor cortex neurons in relation to direction of an intended movement. J. Neurophysiol. 39: 1062-1068.

Tatton, W. G., S. D. Forner, G. L. Gerstein, W. W. Chambers, and C. M. Liu (1975) The effect of post-central cortical lesions on motor responses to sudden upper limb displacements in monkeys. Brain Res. 96: 108-113.

Thomas, J. S., J. Brown, and G. E. Lucier (1977) Influence of task set on muscular responses to arm perturbations in normal subjects and Parkinson patients. Exp. Neurol. 55: 618628.

Tracey, D. J., B. Walmsley, and J. Brinkman (1980) "Longloop" reflexes can be obtained in spinal monkeys. Neurosci. Lett. 18: 59-65. 